\title{
An Analytical Computational Algorithm for Solving a System of Multipantograph DDEs Using Laplace Variational Iteration Algorithm
}

\author{
Mohamed S. M. Bahgat $\mathbb{D}^{1}$ and A. M. Sebaq ${ }^{2,3}$ \\ ${ }^{1}$ Mathematics Department, Faculty of Science, Minia University, Minia, Egypt \\ ${ }^{2}$ Biomedical Engineering Department, Faculty of Engineering, Minia University, Minia, Egypt \\ ${ }^{3}$ Center for Informatics Science, Nile University, Giza, Egypt \\ Correspondence should be addressed to Mohamed S. M. Bahgat; msmbahgat66@hotmail.com
}

Received 15 April 2021; Accepted 19 May 2021; Published 14 June 2021

Academic Editor: Jianguo Yan

Copyright ( 2021 Mohamed S. M. Bahgat and A. M. Sebaq. This is an open access article distributed under the Creative Commons Attribution License, which permits unrestricted use, distribution, and reproduction in any medium, provided the original work is properly cited.

\begin{abstract}
In this research, an approximation symbolic algorithm is suggested to obtain an approximate solution of multipantograph system of type delay differential equations (DDEs) using a combination of Laplace transform and variational iteration algorithm (VIA). The corresponding convergence results are acquired, and an efficient algorithm for choosing a feasible Lagrange multiplier is designed in the solving process. The application of the Laplace variational iteration algorithm (LVIA) for the problems is clarified. With graphics and tables, LVIA approximates to a high degree of accuracy with a few numbers of iterates. Also, computational results of the considered examples imply that LVIA is accurate, simple, and appropriate for solving a system of multipantograph delay differential equations (SMPDDEs).
\end{abstract}

\section{Introduction}

Many physical phenomena are formulated by delay differential equations which are similar to ordinary differential equations, but their development at assured time instant depends on past values. Pantograph equations are one of the most prominent kinds of functional differential equations with proportional delay and the pantograph kind equations have been studied extensively because of the various implementations in which these equations arise. The name of the pantograph is begun from being crafted by Ockendon and Tayler on the gathering of current by the pantograph head of an electric loco; this equation has appeared in many scientific models which is exceedingly applicable in physics, mathematics, engineering, and biology as in astrophysics, population models, probability theory, quantum mechanics, number theory, nonlinear dynamic system, electronic system, cell growth, and so forth (for further, see [1-3] and the references therein). Numerous examinations were done on the estimated arrangement of the referenced condition in the one-dimensional case. As of late, a collocation method based on the Genocchi delay operational matrix and the operational matrix of fractional derivative for solving generalized fractional pantograph equations is given in [4]. Some papers like $[5,6]$ have presented the solution for a system of multipantograph delay differential equations (SMPDDEs) with higher order by two different algorithms. A collocation method based on the Genocchi operational matrix for solving generalized pantograph equations is given in [7]. Then, again, the mathematical resolvability of another form of differential issues can be found in [8-18] and the references therein. The pantograph equation,

$$
\begin{aligned}
y^{\prime}(t) & =\alpha y(t)+f(t, y(\beta t)), \quad 0<\beta<1, \\
y(0) & =y_{0},
\end{aligned}
$$

is one of the important types of DDE that emerge in numerous scientific models which appear in dynamical systems, population studies, electrodynamics, and number theory. In particular, it was used by [3] to research how the 
electric current is composed through the pantograph of an electric locomotive, from where it gets its name. In this research, we are building on our work in [5] to develop the
Laplace variational iteration algorithm (LVIA) for SMPDDEs:

$$
\begin{aligned}
& y_{1}^{\prime}(t)=\alpha_{1,1} y_{1}(t)+\alpha_{1,2} y_{2}(t)+\cdots+\alpha_{1, n} y_{n}(t)+g_{1}\left(t, y_{1}\left(\beta_{1,1} t\right), y_{2}\left(\beta_{1,2} t\right), \ldots, y_{n}\left(\beta_{1, n} t\right)\right) \\
& y_{2}^{\prime}(t)=\alpha_{2,1} y_{1}(t)+\alpha_{2,2} y_{2}(t)+\cdots+\alpha_{2, n} y_{n}(t)+g_{2}\left(t, y_{1}\left(\beta_{2,1} t\right), y_{2}\left(\beta_{2,2} t\right), \ldots, y_{n}\left(\beta_{2, n} t\right)\right) \\
& \quad \vdots \\
& y_{n}^{\prime}(t)=\alpha_{n, 1} y_{1}(t)+\alpha_{n, 2} y_{2}(t)+\cdots+\alpha_{n, n} y_{n}(t)+g_{n}\left(t, y_{1}\left(\beta_{n, 1} t\right), y_{2}\left(\beta_{n, 2} t\right), \ldots, y_{n}\left(\beta_{n, n} t\right)\right),
\end{aligned}
$$

subject to the initial conditions:

$$
y_{i}\left(t_{0}\right)=y_{i, 0}, \quad i=1,2,3, \ldots, n,
$$

where $0=t_{0}<t \leq T, \alpha_{i, j}, y_{i, 0}$ are finite constants, $g_{i}$ are analytical functions such that $0<\beta_{i, j}<1, i, j=1,2, \ldots, n$, which verify all needed requirements for finding a unique solution, and $y_{i}(t), i=1,2, \ldots, n$, are functions that need to be found on the given interval. We were motivated to apply LVIA to find approximate solutions for SMPDDEs. By choosing a suitable value for the initial approximation, LVIA can easily be applied to the given problems. Moreover, the solution and its derivative are usable for each arbitrary point in the interval. LVIA provides a direct scheme for solving the problem with no physically unsuitable assumptions, discretization, linearization, transformation, or perturbation. Generally, in applications of LVIA to IVP (initial value problem) of SMPDDEs, one generally follows the following three proceedings:

(a) Establishment of the correction functional

(b) Identifying the Lagrange multipliers

(c) Defining the initial iteration

LVIA is worth mentioning that the method is capable of decreasing the size of the computational work as compared to the classic methods while yet maintaining the elevation accuracy of the numerical results. The main feature of the variational iteration algorithm over decomposition restraint of Adomian is that the previous algorithm gives the solution of the problem without calculating Adomian's polynomials which requires complex calculations. Accordingly, it is not affected by calculation round-off errors and one is not reputed with the requirement of large PC time and memory. Generally, by using LVIA, one iteration results in an accurate solution if the initial solution is closely chosen. The convergence of the method is a systematical debate in [8]. The remainder of this study is ordered as follows: in Section 2 , we presented the basic idea of LVIA together with the analysis of the method. In Section 3, the symbolic approximate solutions for (2)-(3) can be provided using the extended LVIA. Depending on the above-mentioned, some of the numerical applications are given to explain the adequacy of LVIA in Section 3. The computations show that the approximate solutions can be achieved accurately and efficiently with a few iterations. Finally, Section 4 gives a brief conclusion.

\section{Methodology Basic Ideas of Laplace Variational Iteration Algorithm (LVIA)}

To clarify the basic connotation of LVIA, firstly, we rewrite (2)-(3) as the next form:

$$
Y^{\prime}(t)=\boldsymbol{\alpha} \mathbf{Y}(t)+\mathbf{G}(t, \mathbf{Y}(\boldsymbol{\beta} t))
$$

with the initial conditions:

$$
\mathbf{Y}\left(t_{0}\right)=\mathbf{Y}_{0}
$$

where $\quad Y^{\prime}(t)=\left[y_{1}^{\prime}(t) y_{2}^{\prime}(t) \ldots y_{n}^{\prime}(t)\right]^{T} \in \mathbb{R}^{n}, \quad \mathbf{Y}(t)=$ $\left[y_{1}(t) y_{2}(t) \ldots y_{n}(t)\right]^{T} \in \mathbb{R}^{n}, \quad \alpha, \beta \in \mathbb{R}^{n \times n}, \quad \mathbf{G}=\left[g_{1} g_{2} \ldots\right.$ $\left.g_{n}\right]^{T} \in \mathbb{R}^{n}$ where $g_{i}=g_{i}\left(t, y_{1}\left(\beta_{i, 1} t\right), y_{2}\left(\beta_{i, 2} t\right), \ldots, y_{n}\left(\beta_{i, n} t\right)\right)$, and $\mathbf{Y}_{0}=\left[\begin{array}{llll}y_{1,0} & y_{2,0} & \ldots & y_{n, 0}\end{array}\right]^{T} \in \mathbb{R}^{n}$. The idea of the VIA for (4) is to construct the next correction functional:

$$
\mathbf{Y}_{k+1}(t)=\mathbf{Y}_{k}(t)+\int_{0}^{t} \mu(t, \rho) \mathbf{Y}_{k}^{\prime}(\rho)-\boldsymbol{\alpha} \mathbf{Y}(\rho)-\mathbf{G}(\rho, \mathbf{Y}(\boldsymbol{\beta} \rho)) \mathrm{d} \rho
$$

where $\mu$ is the Lagrange multiplier which has a critical part in this study; it is recognized ideally by variety hypothesis and $\mathbf{Y}_{k}(t), k \geq 0$ is the $n$-th approximate solution order for the exact solution $\mathbf{Y}(t)$, which will be gotten by utilizing starting approximation $\mathbf{Y}(0)$, which is acquired from (5). So, when $n$ tends to infinity, the approximate solution $\mathbf{Y}_{k}(t)$ converges to $\mathbf{Y}(t)$. The numerical procedure of LVIA delineates how LVIA is utilized to approximate the solution of SMPDDEs. The basic steps included are given as follows:

(i) The correction functional is acquired by taking the Laplace transform of (4):

$$
\begin{aligned}
\mathbf{Y}_{k+1}(s)= & \mathbf{Y}_{k}(s)+\mu(s)\left(s \mathbf{Y}_{k}(s)-\mathbf{Y}(0)\right. \\
& +\mathscr{L}\{-\boldsymbol{\alpha} \mathbf{Y}(t)-G(t, \mathbf{Y}(\boldsymbol{\beta} t)\})
\end{aligned}
$$

(ii) where $\mathbf{Y}(s)=\mathscr{L}\{\mathbf{Y}(x)\}=\int_{0}^{\infty} e^{-s x} \mathbf{Y}(x) \mathrm{d} x, \mathscr{L}$ is used to indicate Laplace transform.

(iii) To find the optimal value of $\mu(s)$, take the variation with respect to $\mathbf{Y}_{k}$. Let (7) be stationary with respect to $\mathbf{Y}_{k}$ and $\mathbf{Y}(0)=0$; then, we obtain 


$$
\frac{\delta}{\delta \mathbf{Y}_{k}} \mathbf{Y}_{k+1}(s)=\frac{\delta}{\delta \mathbf{Y}_{k}} \mathbf{Y}_{k}(s)+\frac{\delta}{\delta \mathbf{Y}_{k}}\left(\mu(s)\left(s \mathbf{Y}_{k}(s)-\mathbf{Y}(0)+\mathscr{L}\{-\boldsymbol{\alpha} \mathbf{Y}(t)-G(t, \mathbf{Y}(\boldsymbol{\beta} t)\})\right) .\right.
$$

(iv) Considering the terms $\mathscr{L}\{-\alpha \mathbf{Y}(t)-G t, \mathbf{Y}(\beta t)\}$ as constrained variations, then we get

$$
\delta \mathbf{Y}_{k+1}(s)=\delta \mathbf{Y}_{k}(s)+\mu(s)\left(s \delta \mathbf{Y}_{k}(s)\right) .
$$

(v) From (9), we derive the Lagrange multiplier as

$$
\mu(s)=-\frac{1}{s} .
$$

(vi) By using the inverse of Laplace transform $\mathscr{L}^{-1}$ for (7), we can obtain the succeeding approximations which give

$$
\begin{aligned}
\mathbf{Y}_{k+1}(t) & =\mathbf{Y}_{k}(t)+\mathscr{L}^{-1}\left\{\mu(s)\left(s \mathbf{Y}_{k}(s)-\mathbf{Y}(0)+\mathscr{L}\left\{-\mathbf{\alpha} \mathbf{Y}_{k}(t)-G\left(t, \mathbf{Y}_{k}(\boldsymbol{\beta} t)\right\}\right)\right\}\right. \\
& =\mathscr{L}^{-1}\left(\frac{\mathbf{Y}(0)}{s}\right)+\mathscr{L}^{-1}\left\{\frac{1}{s} \mathscr{L}(\boldsymbol{\alpha} \mathbf{Y}(t)+G(t, \mathbf{Y}(\boldsymbol{\beta} t))\}\right.
\end{aligned}
$$

and initial approximation $\mathbf{Y}_{0}(t)$ can be determined by

$$
\begin{aligned}
\mathbf{Y}_{0}(t) & =\mathscr{L}^{-1}\left(\frac{\mathbf{Y}_{0}}{s}+\cdots+\frac{\mathbf{Y}^{(k-1)}(0)}{s^{k}}\right) \\
& =\mathbf{Y}(0)+Y^{\prime}(0) t+\cdots+\frac{\mathbf{Y}^{(k-1)}(0) t^{k-1}}{(k-1) !} .
\end{aligned}
$$

Beginning with an initial approximation $\mathbf{Y}_{0}(t)$, we obtain the sequential approximations, and the exact solution can be acquired by using

$$
\mathbf{Y}(t)=\lim _{k \longrightarrow \infty} \mathbf{Y}_{k}(t)
$$

Now, we display that the sequence $\left\{\mathbf{Y}_{k}(t)\right\}_{k=1}^{\infty}$ given by (9) with $\mathbf{Y}_{0}(t)=\mathbf{Y}_{0}$ converges to the exact solution of (4)(5). To do this, we declare the following theorem.

Theorem 1 (see [9]). Let $\mathbf{Y}(t) \in\left(C^{1}(\mathbb{R})\right)^{n}, t \in \mathbb{R}=[0, T]$ be the exact solution of $(4)$ and $\mathbf{Y}_{n}(t) \in\left(C^{1}(\mathbb{R})\right)^{k}$ be the solution of the sequence

$$
\begin{aligned}
\mathbf{Y}_{k+1}(t) & =\mathbf{Y}_{k}(t)+\mathscr{L}^{-1}\left\{\mu ( s ) \left(s \mathbf{Y}_{k}(s)-\mathbf{Y}(0)+\mathscr{L}\left\{-\boldsymbol{\alpha} \mathbf{Y}_{k}(t)-G\left(t, \mathbf{Y}_{k}(\boldsymbol{\beta} t)\right\}\right\}\right.\right. \\
& =\mathscr{L}^{-1}\left(\frac{\mathbf{Y}(0)}{s}\right)+\mathscr{L}^{-1}\left\{\frac{1}{s} \mathscr{L}(\boldsymbol{\alpha} \mathbf{Y}(t)+G(t, \mathbf{Y}(\boldsymbol{\beta} t))\}\right.
\end{aligned}
$$

with the initial approximation $\mathbf{Y}_{0}(t)$.

If $\operatorname{Ext}^{k}(t)=\mathbf{Y}(t)-\mathbf{Y}_{k}(t)$ and $\left\|(\mathrm{d} / \mathrm{d} t) \operatorname{Ext}^{k}(t)\right\|_{2} \leq$ $\left\|\operatorname{Ext}^{k}(t)\right\|_{2}$, then the functional sequence $\left\{\mathbf{Y}_{k}(t)\right\}_{k=1}^{\infty}$ defined by the above sequence converges to $\mathbf{Y}(t)$.

Proof. To prove this theorem, we follow the same proof of Theorem 1 in [9].

The accompanying considerable definitions given underneath are needed for this work and furthermore are needed for the convergence analysis in the next corollary.

Corollary 1. Let $y_{i, k}(t), i=1,2, \ldots, m$ and $k=1,2, \ldots, n$, be series for any iteration $k$; then the LVIA will attain approximately to the exact solution of (2)-(3). Now, we report some types of errors which are as follows: (a) Residual error $\operatorname{Res}_{i}^{k}(t)$ defined by $\operatorname{Res}_{i}^{k}(t)=\mid y_{i, k}{ }^{\prime}(t)-$ $\alpha_{i, 1} y_{1, k}(t)-\alpha_{i, 2} y_{2, k}(t)-\cdots-\alpha_{i, m} y_{m, k}(t)-g_{i}\left(t, y_{1, k}\right.$ $\left.\left(\beta_{i, 1} t\right), y_{2, k}\left(\beta_{i, 2} t\right), \ldots, y_{m, k}\left(\beta_{i, m} t\right)\right) \mid$

(b) Exact error (absolute error) Ext $t_{i}^{k}$ which is defined by $E x t_{i}^{k}=\left|y_{i, \text { exact }}(t)-y_{i, k}(t)\right|$

(c) Relative error Rel $l_{i}^{k}$, which is defined by $\operatorname{Rel}_{i}^{k}=$ $\left(\left|y_{i, \text { exact }}(t)-y_{i, k}(t)\right| /\left|y_{i, \text { exact }}(t)\right|\right)$

(d) Consecutive error $\mathrm{Con}_{i}^{k}$, which is defined by $\mathrm{Con}_{i}^{k}=\left|y_{i, k+1}(t)-y_{i, k}(t)\right|$

\section{Applications and Numerical Discussion}

In this section, six numerical examples are given to illustrate the accuracy and the convergence of LVIA which is described in Section 2. In contrast, numerical results show that this method gives a good approximation to the exact 
solution for all possible values of $t$, while the accuracy is in continuous increase by using only a few approximations. For comparison purposes, the solution intervals of problems are chosen generally the same as those in the references. Throughout this research, all the symbolical and computations results used Maple 18.0 software program.

Example 1. Regarding the SMPDDEs [19],

$$
\begin{array}{ll}
y_{1}^{\prime}(t)-y_{1}(t)+y_{2}(t)-y_{1}\left(\frac{t}{2}\right)=f_{1}(t), & \\
y_{2}^{\prime}(t)+y_{1}(t)+y_{2}(t)+y_{2}\left(\frac{t}{2}\right)=f_{2}(t), &
\end{array}
$$

subject to the initial conditions:

$$
\begin{aligned}
& y_{1}(0)=1, \\
& y_{2}(0)=1,
\end{aligned}
$$

where $f_{1}(t)=e^{-t}-e^{(t / 2)}, f_{2}(t)=e^{t}+e^{-(t / 2)}$, with the exact solutions $y_{1}(t)=e^{t}$ and $y_{2}(t)=e^{-t}$.

We apply LVIA for solving system (15) and (16); we begin with selecting the initial conditions of the approximations such as $y_{1,0}(t)=y_{1}(0)=1$ and $y_{2,0}(t)=y_{2}(0)=1$. Then, let us seek the approximate solutions $y_{1, k}(t)$ and $y_{2, k}(t)$ where $k$ is a positive integer number greater than or equal to zero. By taking $\mathscr{L}$ for system (15)-(16), we obtain

$$
\begin{aligned}
& s \bar{y}_{1}(s)-y_{1}(0)=\mathscr{L}\left\{-y_{2}(t)+y_{1}(t)+y_{1}\left(\frac{t}{2}\right)+f_{1}(t)\right\}, \\
& s \bar{y}_{2}(s)-y_{2}(0)=\mathscr{L}\left\{-y_{2}(t)-y_{1}(t)-y_{2}\left(\frac{t}{2}\right)+f_{2}(t)\right\} .
\end{aligned}
$$

The iteration formulas thus are

$$
\begin{aligned}
& \bar{y}_{1, k+1}(s)=\bar{y}_{1, k}(s)+\mu(s)\left\{s \bar{y}_{1, k}(s)-y_{1,0}(0)-\mathscr{L}\left\{y_{1, k}(t)-y_{2, k}(t)+y_{1, k}\left(\frac{t}{2}\right)+f_{1}(t)\right\}\right\}, \\
& \bar{y}_{2, k+1}(s)=\bar{y}_{2, k}(s)+\mu(s)\left\{s \bar{y}_{2, k}(s)-y_{2,0}(0)-\mathscr{L}\left\{-y_{1, k}(t)-y_{2, k}(t)-y_{2, k}\left(\frac{t}{2}\right)+f_{2}(t)\right\}\right\},
\end{aligned}
$$

with the Lagrange multiplier $\mu(s)=-(1 / s)$, and by taking $\mathscr{L}^{-1}$, we obtain

$$
\begin{aligned}
y_{1, k+1}(t) & =y_{1, k}(t)-\mathscr{L}^{-1}\left\{\frac{1}{s}\left\{s \bar{y}_{1, k}(s)-y_{1,0}(0)-\mathscr{L}\left\{y_{1, k}(t)-y_{2, k}(t)+y_{1, k}\left(\frac{t}{2}\right)+f_{1}(t)\right\}\right\}\right\} \\
& =\mathscr{L}^{-1}\left[\frac{1}{s}\right]+\mathscr{L}^{-1}\left\{\frac{1}{s}\left\{\mathscr{L}\left\{y_{1, k}(t)-y_{2, k}(t)+y_{1, k}\left(\frac{t}{2}\right)+f_{1}(t)\right\}\right\}\right\}, \\
y_{2, k+1}(t) & =y_{2, k}(t)-\mathscr{L}^{-1}\left\{\frac{1}{s}\left\{s \bar{y}_{2, k}(s)-y_{2,0}(0)-\mathscr{L}\left\{y_{1, k}(t)-y_{2, k}(t)-y_{2, k}\left(\frac{t}{2}\right)+f_{2}(t)\right\}\right\}\right\} \\
& =\mathscr{L}^{-1}\left[\frac{1}{s}\right]+\mathscr{L}^{-1}\left\{\frac{1}{s}\left\{\mathscr{L}\left\{-y_{1, k}(t)-y_{2, k}(t)-y_{2, k}\left(\frac{t}{2}\right)+f_{2}(t)\right\}\right\}\right\} .
\end{aligned}
$$

Therefore,

$$
\begin{aligned}
& y_{1, k+1}(t)=\mathscr{L}^{-1}\left\{\left(\frac{1}{s}\right)\left\{\mathscr{L}\left\{y_{1, k}(t)-y_{2, k}(t)+y_{1, k}\left(\frac{t}{2}\right)+f_{1}(t)\right\}\right\}\right\}+1 \\
& y_{2, k+1}(t)=\mathscr{L}^{-1}\left\{\left(\frac{1}{s}\right)\left\{\mathscr{L}\left\{-y_{1, k}(t)-y_{2, k}(t)-y_{2, k}\left(\frac{t}{2}\right)+f_{2}(t)\right\}\right\}\right\}+1
\end{aligned}
$$

with the initial iteration $y_{1,0}(t)=y_{1}(0)=1$, $y_{2,0}(t)=y_{2}(0)=1$. Now, in order to obtain the first approximation $y_{1,1}(t)$ and $y_{2,1}(t)$ of the LVIA solution for system (15) and (16), we put $k=0$ through (20) to get 


$$
\begin{aligned}
& y_{1,1}(t)=4-e^{-t}-2 e^{(t / 2)}+t \\
& y_{2,1}(t)=2-2 e^{-(t / 2)}-3 t+e^{t}
\end{aligned}
$$

by using the first approximation $y_{1,1}(t)$ and $y_{2,1}(t)$; then, the second approximation $y_{1,2}$ and $y_{2,2}(t)$ of the LVIA solution for system (15)-(16) can be written as follows:

$$
\begin{aligned}
& y_{1,2}(t)=\frac{18-8 e^{(t / 4)}-6 e^{(t / 2)}-2 e^{-(t / 2)}+6 t-e^{t}+9}{4 t^{2}}, \\
& y_{2,2}(t)=\frac{14-8 e^{-(t / 4)}-e^{-t}+2 e^{(t / 2)}-6 e^{-(t / 2)}-8 t+7}{4 t^{2}},
\end{aligned}
$$

and by proceeding with the comparable style, the third and the fourth iterations for the LVIA solution of (15)-(16) lead to the following outcomes:

$$
\begin{aligned}
y_{1,3}(t)= & \frac{176-24 e^{-(t / 4)}-2 e^{-t}-56 e^{(t / 4)}-20 e^{(t / 2)}-8 e^{-(t / 2)}+\left(17 t^{3} / 48\right)+22 t-64 e^{(t / 8)}-e^{t}+17}{2 t^{2}}, \\
y_{2,3}(t)= & 108-56 e^{-(t / 4)}-e^{-t}+24 e^{(t / 4)}+8 e^{(t / 2)}-20 e^{-(t / 2)}-\frac{71 t^{3}}{48}-46 t-64 e^{-(t / 8)}+2 e^{t}+3 t^{2}, \\
y_{1,4}(t)= & 2884-96 e^{-(t / 4)}-1024 e^{(t / 16)}-400 e^{(t / 4)}-60 e^{(t / 2)}-20 e^{-(t / 2)}+\frac{61 t^{3}}{24}+244 t \\
& +\frac{721 t^{4}}{1536}-320 e^{-(t / 8)}-960 e^{(t / 8)}-3 e^{t}+\frac{79 t^{2}}{2}, \\
& +\frac{503 t^{4}}{1536}-960 e^{-(t / 8)}+320 e^{(t / 8)}+\frac{47 t^{2}}{2} .
\end{aligned}
$$

To clarify the convergence of the solution of the proposed method $y_{i, k}(t)$ to the exact solution $y_{i}(t), i=1,2$, w.r.t. the number of iterations $k$ of the solutions, we set numerical values graphically. Figure 1(a) shows the exact solution $y_{i}(t), i=1,2, t \in[0,3]$, and $y_{i, k}(t), k=3,5,7,9$, $i=1,2$, respectively. These plots detect that LVIA is an effective and appropriate method to solve SMPDDEs with fewer calculations and numbers of iterations. Figure 1(b) illustrates the efficiency of the method whether it has an exact solution or not by using the residual error values which shows that the residual error $\operatorname{Res}_{i}^{k}(t)$ decreases as $k$ increases. Table 1 shows the CPU times of the present method for Example 1 to get $10^{\text {th }}$-order approximate symbolic solutions with variable $t$. The accuracy of the method can be spotted with the numerical results and by comparing the absolute errors of LVIA for $k=4,5,6$ and the Laplace decomposition algorithm (LDA) [19] is given in Table 2. It is obvious that, from this table, the approximate solutions are proven to be similar to the exact solution for all likely values of $t$ in $[0,1]$.
Example 2. Consider SMPDDEs [20]:

$$
\begin{aligned}
& y_{1}^{\prime}(t)-\sin (t) y_{1}\left(\frac{t}{2}\right)-\cos (t) y_{2}\left(\frac{t}{2}\right)=f_{1}(t), \\
& y_{2}^{\prime}(t)+\cos (t) y_{1}\left(\frac{t}{2}\right)-\sin (t) y_{2}\left(\frac{t}{2}=f_{2}(t)\right),
\end{aligned}
$$

subject to the initial conditions:

$$
\begin{aligned}
& y_{1}(0)=0, \\
& y_{2}(0)=1,
\end{aligned}
$$

where $f_{1}(t)=\cos (t)-\cos (t / 2), f_{2}(t)=-\sin (t)-\sin (t / 2)$ with the exact solutions $y_{1}(t)=\sin (t)$ and $y_{2}(t)=\cos (t)$. By the same procedures of Example 1, we apply the LVIA approach for solving (24)-(25), according to (11). Table 3 shows the CPU times of the LVIA for Example 2 to get $10^{\text {th }}$ order approximate symbolic solutions with variable $t$. To 


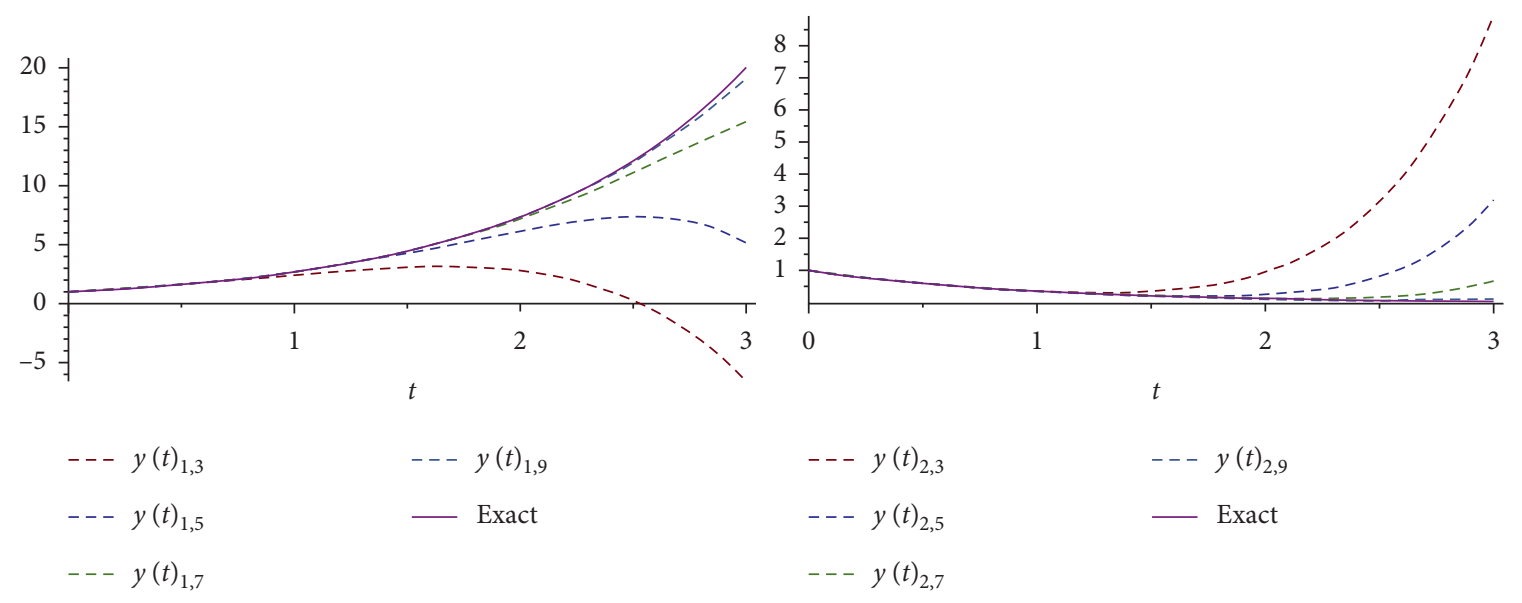

(a)


(b)

Figure 1: (a) Comparison between $y_{i, k}(t), k=3,5,7,9, i=1,2$ solutions of the LVIA and the exact solution for Example 1. (b) The residual error $\operatorname{Res}_{i}^{k}(t), i=1,2$ of LVIA solutions of (13) at $k=4,6,8$, respectively, for Example 1 .

TABle 1: CPU times (per second) of LVIA for Example 1.

\begin{tabular}{lcccccccccc}
\hline$k$ & 1 & 2 & 3 & 4 & 5 & 6 & 7 & 8 & 9 & 10 \\
\hline CPU & 0.143 & 0.254 & 0.415 & 0.544 & 0.744 & 0.936 & 1.158 & 1.442 & 1.720 & 2.078 \\
\hline
\end{tabular}

TABle 2: Comparison of the absolute errors $\left(\operatorname{Ext}_{i}^{k}, i=1,2\right)$ for Example 1.

\begin{tabular}{|c|c|c|c|c|c|}
\hline \multirow{2}{*}{$t$} & \multirow{2}{*}{ Exact solution $y_{1}(t)$} & \multicolumn{2}{|c|}{$y_{1, k}(t)$ LDA [19] } & \multicolumn{2}{|c|}{$y_{1, k}(t)$ (presented method) } \\
\hline & & $k=4$ & $k=6$ & $k=4$ & $k=5$ \\
\hline 0.2 & 1.2214027581602 & $1.210 \times 10^{-5}$ & $1.210 \times 10^{-5}$ & $1.576 \times 10^{-5}$ & $2.276 \times 10^{-6}$ \\
\hline 0.4 & 1.4918246976413 & $4.238 \times 10^{-4}$ & $3.170 \times 10^{-6}$ & $5.057 \times 10^{-4}$ & $9.470 \times 10^{-5}$ \\
\hline 0.6 & 1.8221188003905 & $3.499 \times 10^{-3}$ & $5.583 \times 10^{-5}$ & $3.981 \times 10^{-3}$ & $8.488 \times 10^{-5}$ \\
\hline 0.8 & 2.2255409284925 & $1.594 \times 10^{-2}$ & $4.460 \times 10^{-4}$ & $1.736 \times 10^{-3}$ & $4.721 \times 10^{-4}$ \\
\hline \multirow[t]{3}{*}{1.00} & 2.7182818284591 & $5.236 \times 10^{-2}$ & $2.259 \times 10^{-3}$ & $5.484 \times 10^{-3}$ & $1.810 \times 10^{-4}$ \\
\hline & & \multicolumn{2}{|c|}{$y_{2, k}(t)$ LDA [19] } & \multicolumn{2}{|c|}{$y_{2, k}(t)$ (presented metdod) } \\
\hline & Exact solution $y_{2}(t)$ & $k=4$ & $k=6$ & $k=4$ & $k=5$ \\
\hline 0.2 & 0.8187307530780 & $5.219 \times 10^{-5}$ & $7.807 \times 10^{-8}$ & $1.725 \times 10^{-5}$ & $8.753 \times 10^{-6}$ \\
\hline 0.4 & 0.6703200460356 & $1.668 \times 10^{-3}$ & $1.310 \times 10^{-5}$ & $5.739 \times 10^{-4}$ & $1.407 \times 10^{-5}$ \\
\hline 0.6 & 0.5488116360940 & $1.266 \times 10^{-2}$ & $2.227 \times 10^{-4}$ & $4.271 \times 10^{-3}$ & $5.764 \times 10^{-5}$ \\
\hline 0.8 & 0.4493289641172 & $5.338 \times 10^{-2}$ & $1.668 \times 10^{-3}$ & $1.765 \times 10^{-2}$ & $2.080 \times 10^{-4}$ \\
\hline 1.0 & 0.3678794411714 & $1.632 \times 10^{-1}$ & $7.956 \times 10^{-3}$ & $5.284 \times 10^{-2}$ & $3.014 \times 10^{-4}$ \\
\hline
\end{tabular}


TABLE 3: CPU times (per second) of LVIA for Example 2.

\begin{tabular}{lcccccccccc}
\hline$k$ & 1 & 2 & 3 & 4 & 5 & 6 & 7 & 8 & 9 & 10 \\
\hline CPU & 0.153 & 0.311 & 0.571 & 0.909 & 01.523 & 2.722 & 2.997 & 3.243 & 3.508 & 3.792 \\
\hline
\end{tabular}

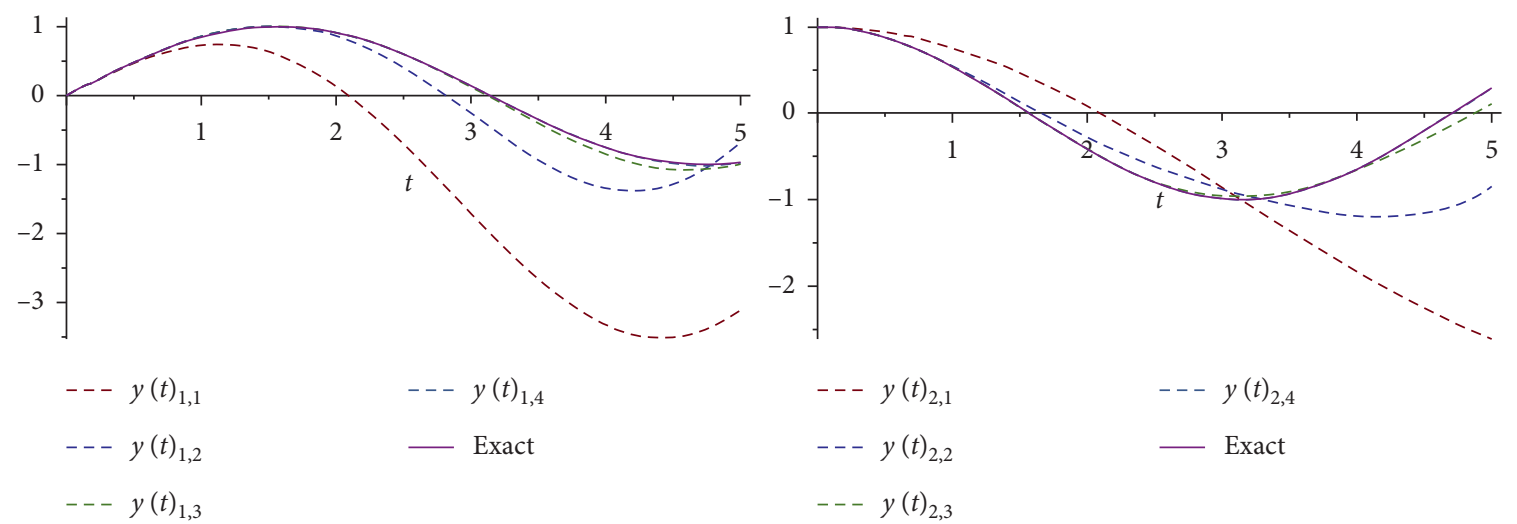

Figure 2: Plots of the LVIA solutions $y_{i, k}(t), i=1,2, k=1,2,3,4$, respectively, and the exact solution $y_{i}(t), i=1,2$, of Example 2 on [0,5], where $y_{i}(t)$ is represented by a straight line and $y_{i, k}(t)$ is represented by a dashed line.

TABLE 4: Error analysis.

\begin{tabular}{|c|c|c|c|c|}
\hline$t$ & Absolute error $\mathrm{Ext}_{1}^{10}$ & Relative error $\operatorname{Rel}_{1}^{10}$ & Consecutive error $\operatorname{Con}_{1}^{10}$ & Residual error $\operatorname{Res}_{1}^{10}(t)$ \\
\hline \multicolumn{5}{|c|}{ Error analysis of $y_{1}(t)$ for Example 2 on $[0,1]$} \\
\hline 0.2 & $1.34 \times 10^{-32}$ & $6.77 \times 10^{-32}$ & $1.34 \times 10^{-32}$ & $7.32 \times 10^{-31}$ \\
\hline 0.4 & $2.29 \times 10^{-29}$ & $5.87 \times 10^{-29}$ & $2.29 \times 10^{-29}$ & $5.99 \times 10^{-28}$ \\
\hline 0.6 & $1.36 \times 10^{-27}$ & $2.401 \times 10^{-27}$ & $1.35 \times 10^{-27}$ & $2.13 \times 10^{-26}$ \\
\hline 0.8 & $1.39 \times 10^{-26}$ & $1.94 \times 10^{-26}$ & $1.39 \times 10^{-26}$ & $9.50 \times 10^{-26}$ \\
\hline 1.0 & $6.61 \times 10^{-26}$ & $7.86 \times 10^{-26}$ & $6.62 \times 10^{-26}$ & $1.87 \times 10^{-24}$ \\
\hline \multicolumn{5}{|c|}{ Error analysis of $y_{2}(t)$ for Example 4 on $[0,1]$} \\
\hline 0.2 & $4.66 \times 10^{-33}$ & $4.75 \times 10^{-33}$ & $4.66 \times 10^{-33}$ & $2.79 \times 10^{-31}$ \\
\hline 0.4 & $1.80 \times 10^{-29}$ & $1.95 \times 10^{-29}$ & $1.80 \times 10^{-29}$ & $5.33 \times 10^{-28}$ \\
\hline 0.6 & $2.11 \times 10^{-27}$ & $2.56 \times 10^{-27}$ & $2.11 \times 10^{-27}$ & $5.00 \times 10^{-26}$ \\
\hline 0.8 & $2.76 \times 10^{-26}$ & $8.27 \times 10^{-26}$ & $5.76 \times 10^{-26}$ & $8.14 \times 10^{-26}$ \\
\hline 1.0 & $6.83 \times 10^{-25}$ & $1.26 \times 10^{-24}$ & $8.83 \times 10^{-25}$ & $7.39 \times 10^{-24}$ \\
\hline
\end{tabular}

clarify the convergence of the solution of the proposed method $y_{i, k}(t)$ to the exact solutions $y_{i}(t), i=1,2$, with respect to the iteration number of the solution, we set numerical values graphically. Figure 2 shows the exact solution $y_{i}(t), i=1,2, t \in[0,5]$, and some iterated approximations $y_{i, k}(t), k=1,2,3,4, i=1,2$, respectively. This graph and the results obtained in Table 4 detected that the LVIA is a very accurate and effective method to solve SMPDDEs with fewer computational and iteration steps.

Example 3. Consider the two-dimensional nonlinear pantograph equations [19]:

$$
\begin{array}{ll}
y_{1}^{\prime}(t)+y_{1}(t)+e^{-t} \cos \left(\frac{t}{2}\right) y_{2}\left(\frac{t}{2}\right)+2 e^{(-3 / 4) t} \cos \left(\frac{t}{2}\right) \sin \left(\frac{t}{4}\right) y_{1}\left(\frac{t}{4}\right)=0, & 0 \leq t \leq 1, \\
y_{2}^{\prime}(t)-e^{t}\left(y_{1}\left(\frac{t}{2}\right)\right)^{2}+\left(y_{2}\left(\frac{t}{2}\right)\right)^{2}=0, &
\end{array}
$$

the initial conditions in which the system subjected to

$$
\begin{aligned}
& y_{1}(0)=1, \\
& y_{2}(0)=0 .
\end{aligned}
$$

The exact solutions are $y_{1}(t)=e^{-t} \cos (t)$ and $y_{2}(t)=$ $\sin (t)$. By the same procedures of Example 1, we apply LVIA for solving (26)-(27), according to (11). To clarify the convergence of $y_{i, k}(t)$ to $y_{i}(t), i=1,2$, with respect to the iteration number of the solution, we present numerical results of Example 3 graphically where Figures 3(a) and 3(b) show the exact solution and the approximate solution of LVIA for $y_{i, k}(t), i=1,2, k=1,2,3$, respectively. These plots clarify that LVIA is a good accurate method for 


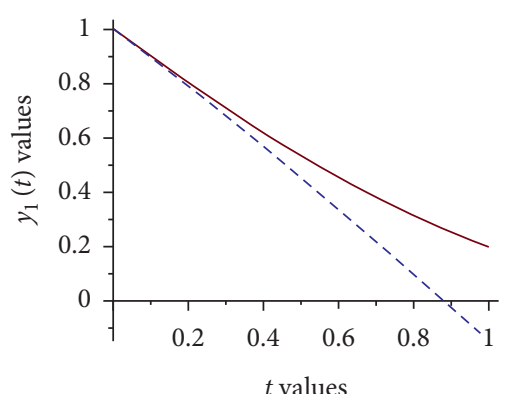

Exact solution plot
Approximate solution plot for
$y_{1,1}(t)$

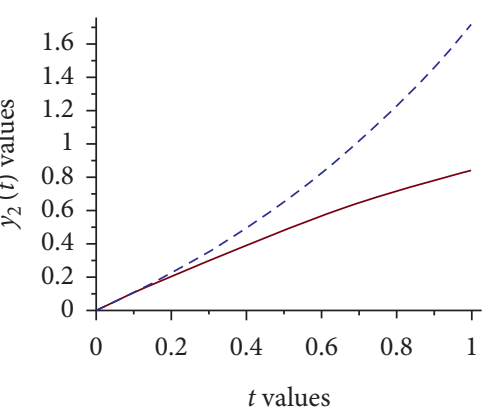

_ Exact solution plot

— Approximate solution plot for $y_{2,1}(t)$

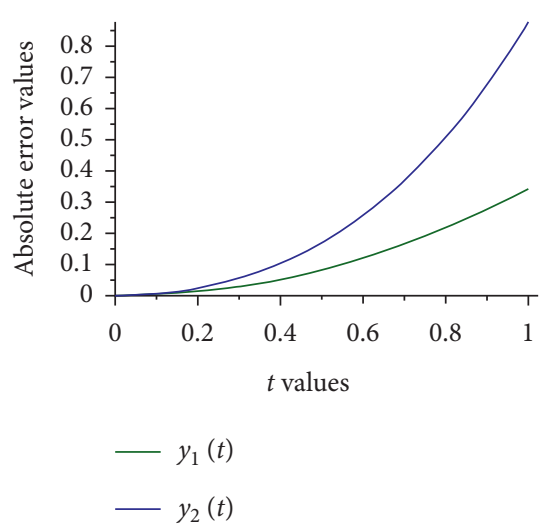

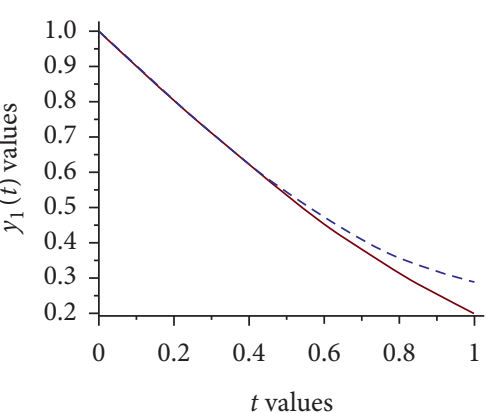

_ Exact solution plot

_ Approximate solution plot for $y_{1,1}(t)$

(a)

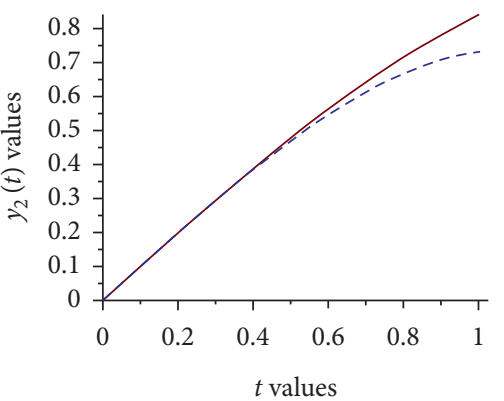

_ Exact solution plot

— Approximate solution plot for $y_{2,2}(t)$

(b)
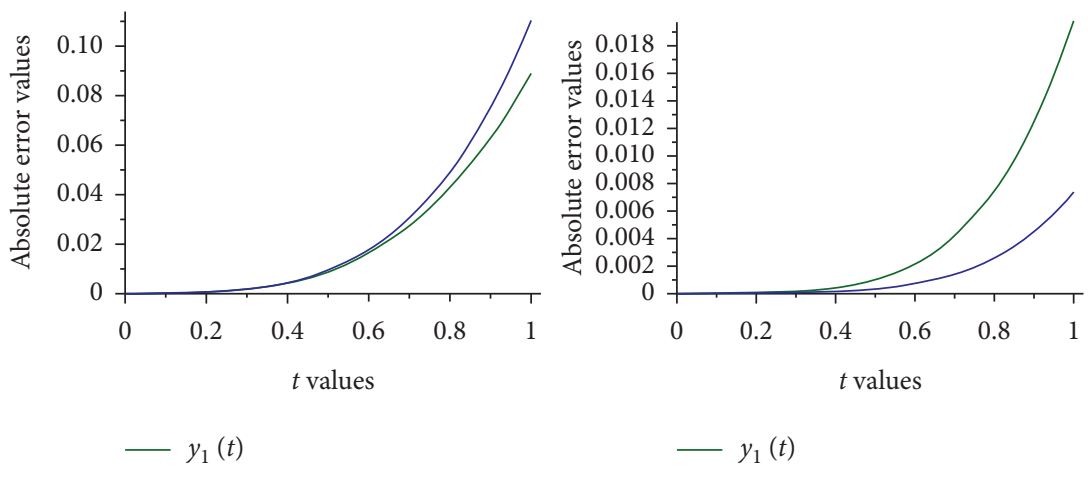

$-y_{1}(t)$

$-y_{2}(t)$

(c)
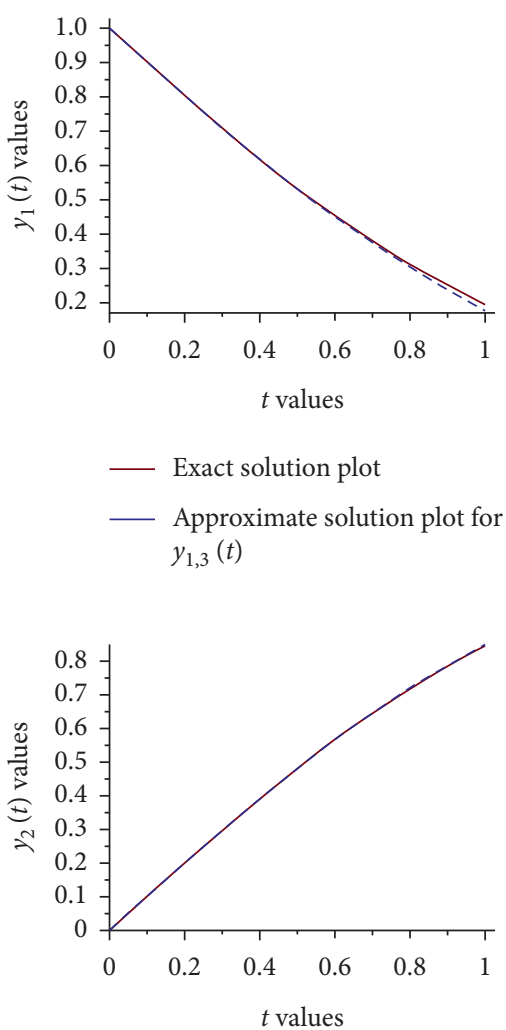

_ Exact solution plot

— Approximate solution plot for $y_{2,3}(t)$

FIgURE 3: (a) Comparison between $y_{1, k}(t), k=1,2,3$ solutions of the LVIA and $y_{1}(t)$ for Example 3. (b) Comparison between $y_{2, k}(t), k=$ 1, 2, 3 of LVIA and the exact solution $y_{2}(t)$ for Example 3. (c) Comparison of Ext ${ }_{i}^{k}$ for $k=1,2,3$, respectively, for $y_{i, k}(t), i=1,2$ of Example 3 .

solving such SMPDDEs with fewer calculations and numbers of iterations. Also, Figure 3(c) displays the absolute errors obtained by LVIA for $k=1,2,3$ of (26)-(27). From Figure 4(c), the approximate solutions at $k=1,2,3$ are identical to the exact solution for all values of $t$ in $[0,1]$. These plots clarify that LVIA is an efficient method for solving such a system with a few iterations. We note that the error decreases while the value $k$ increases.
Example 4. Consider the 2-dimensional nonlinear SMPDDEs [21]:

$$
\begin{aligned}
& y_{1}^{\prime}(t)-y_{1}^{\prime}(t-1)-4 y_{2}(t)=0, \\
& y_{2}^{\prime}(t)-y_{1}(t)+y_{1}(t-1)=0,
\end{aligned}
$$

subject to the initial functions: 


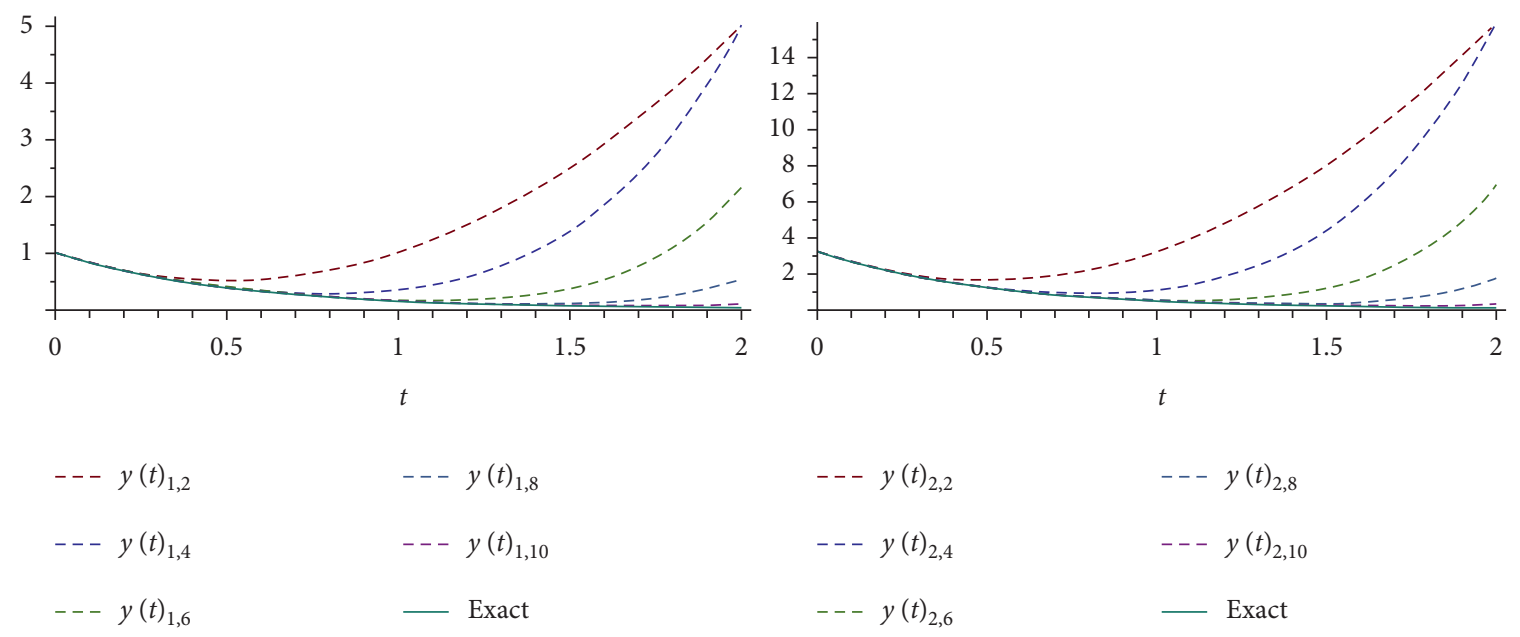

FIgURE 4: Plots of the LVIA solutions $y_{i, k}(t), i=1,2, k=2,4,6,8,10$, respectively, and the exact solution $y_{i}(t), i=1,2$, of Example 4 on $[0,2]$, where $y_{i}(t)$ is represented by a straight line and $y_{i, k}(t)$ is represented by a dashed line.

$$
\begin{aligned}
& y_{1}(t)=e^{-2 t}, \\
& y_{2}(t)=\frac{1}{2}\left(e^{-2(t-1)}-e^{-2 t}\right), \quad-1 \leq t \leq 0 .
\end{aligned}
$$

The exact solutions are $y_{1}(t)=e^{-2 t}$ and $(1 / 2)\left(e^{-2(t-1)}-e^{-2 t}\right)$. By using the given initial functions and also using the same procedures of Example 1, we apply the LVIA approach for solving (28)-(29), according to (11). Without loss of generality, we will test the accuracy of LVIA for Example 4 using error analysis of $y_{i}(t), i=1,2, t \in[0,1]$ for (28)-(29) with a step size of 0.2 as well as comparison among the absolute errors, relative errors, consecutive errors, and residual errors of $10^{\text {th }}$-order approximate LVIA solutions shown in Table 5 . Also, to illustrate the convergence of $y_{i, k}(t)$ to $y_{i}(t), i=1,2$, with respect to the order of $k$ for the solutions, we ready numerical results of Example 4 graphically where Figure 4 shows the exact solutions $y_{i}(t), i=1,2$, and approximate solutions $y_{i, k}(t), i=1,2$, and $k=2,4,6,8,10$, respectively. These plots clarify that LVIA is an efficient method for solving such a system with a few iterations. We note that the error decreases while the value $k$ increases. The results show that the LVIA provides us with the precise approximate solutions of (28)-(29). Moreover, we can control the error also by evaluating more iterations.
Example 5. Consider 3-dimensional SMPDDEs [22]:

$$
\begin{aligned}
y_{1}^{\prime}(t)-2 y_{2}\left(\frac{t}{2}\right)-y_{3}(t) & =f_{1}(t), \\
y_{2}^{\prime}(t)+2\left(y_{3}\left(\frac{t}{2}\right)\right)^{2} & =f_{2}(t), \quad t \geq 0 \\
y_{3}^{\prime}(t)-y_{2}(t)+y_{1}(t) & =f_{3}(t)
\end{aligned}
$$

subject to the initial conditions:

$$
\begin{aligned}
& y_{1}(0)=-1, \\
& y_{2}(0)=0, \\
& y_{3}(0)=0,
\end{aligned}
$$

where $f_{1}(t)=-t \cos ((1 / 2) t), f_{2}(t)=1-t \sin (t) \quad$ and $f_{3}(t)=-t \cos (t)$ with the exact solutions $y_{1}(t)=-\cos (t)$, $y_{2}(t)=t \cos (t)$, and $y_{3}(t)=\sin (t)$. Using the LVIA method for solving (30)-(31), at first we select the initial values of the approximations such as $y_{1,0}=-1$ and $y_{2,0}(0)=y_{3,0}(0)=0$; then, let us seek the approximate solutions $y_{1, k}, y_{2, k}$, and $y_{3, k}$. Taking $\mathscr{L}$ for (30)-(31), we obtain

$$
\begin{aligned}
& s \bar{y}_{1}(s)-y_{1,0}(0)=\mathscr{L}\left\{2 y_{2}\left(\frac{t}{2}\right)+y_{3}(t)+f_{1}(t)\right\}, \\
& s \bar{y}_{2}(s)-y_{2,0}(0)=\mathscr{L}\left\{-2\left(y_{3}\left(\frac{t}{2}\right)\right)^{2}+f_{2}(t)\right\} \\
& s \bar{y}_{3}(s)-y_{3,0}(0)=\mathscr{L}\left\{y_{2}(t)-y_{1}(t)+f_{3}(t)\right\} .
\end{aligned}
$$


TABle 5: Error analysis.

\begin{tabular}{|c|c|c|c|c|}
\hline$t$ & Absolute error $\mathrm{Ext}_{1}^{10}$ & Relative error $\operatorname{Rel}_{1}^{10}$ & Consecutive error $\operatorname{Con}_{1}^{10}$ & Residual error $\operatorname{Res}_{1}^{10}(t)$ \\
\hline \multicolumn{5}{|c|}{ Error analysis of $y_{1}(t)$ for Example 4 on $[0,1]$} \\
\hline 0.2 & $1.02 \times 10^{-12}$ & $1.52 \times 10^{-12}$ & $8.00 \times 10^{-13}$ & $4.28 \times 10^{-11}$ \\
\hline 0.4 & $2.02 \times 10^{-9}$ & $4.49 \times 10^{-9}$ & $1.15 \times 10^{-9}$ & $2.94 \times 10^{-8}$ \\
\hline 0.6 & $1.69 \times 10^{-7}$ & $5.61 \times 10^{-7}$ & $6.03 \times 10^{-8}$ & $9.13 \times 10^{-7}$ \\
\hline 0.8 & $3.88 \times 10^{-6}$ & $1.92 \times 10^{-5}$ & $5.44 \times 10^{-7}$ & $3.19 \times 10^{-6}$ \\
\hline 1.0 & $4.40 \times 10^{-5}$ & $3.24 \times 10^{-4}$ & $3.38 \times 10^{-6}$ & $8.45 \times 10^{-5}$ \\
\hline \multicolumn{5}{|c|}{ Error analysis of $y_{2}(t)$ for Example 4 on $[0,1]$} \\
\hline 0.2 & $3.25 \times 10^{-12}$ & $1.52 \times 10^{-12}$ & $3.23 \times 10^{-12}$ & $1.77 \times 10^{-10}$ \\
\hline 0.4 & $6.44 \times 10^{-9}$ & $4.49 \times 10^{-9}$ & $6.38 \times 10^{-9}$ & $1.74 \times 10^{-7}$ \\
\hline 0.6 & $5.40 \times 10^{-7}$ & $5.61 \times 10^{-7}$ & $5.32 \times 10^{-7}$ & $9.66 \times 10^{-6}$ \\
\hline 0.8 & $1.24 \times 10^{-5}$ & $1.92 \times 10^{-5}$ & $1.21 \times 10^{-5}$ & $1.65 \times 10^{-4}$ \\
\hline 1.0 & $1.40 \times 10^{-4}$ & $3.24 \times 10^{-4}$ & $1.37 \times 10^{-4}$ & $1.48 \times 10^{-3}$ \\
\hline
\end{tabular}

The iteration formulas thus are

$$
\begin{aligned}
& \bar{y}_{1, k+1}(s)=\bar{y}_{1, k}(s)+\mu(s)\left\{s \bar{y}_{1, k}(s)-y_{1,0}(0)-\mathscr{L}\left\{2 y_{2, k}\left(\frac{t}{2}\right)+y_{3, k}(t)+f_{1}(t)\right\}\right\}, \\
& \bar{y}_{2, k+1}(s)=\bar{y}_{2, k}(s)+\mu(s)\left\{s \bar{y}_{2}(s)-y_{2,0}(0)-\mathscr{L}\left\{-2\left(y_{3, k}\left(\frac{t}{2}\right)\right)^{2}+f_{2}(t)\right\}\right\}, \\
& \bar{y}_{3, k+1}(s)=\bar{y}_{3, k}(s)+\mu(s)\left\{s \bar{y}_{3, k}(s)-y_{3,0}(0)-\mathscr{L}\left\{y_{2, k}(t)-y_{1, k}(t)+f_{3}(t)\right\}\right\},
\end{aligned}
$$

with the Lagrange multiplier $\mu(s)=-(1 / s)$, and by taking $\mathscr{L}^{-1}$, we obtain

$$
\begin{aligned}
& y_{1, k+1}(t)=y_{1, k}(t)-\mathscr{L}^{-1}\left\{\frac{1}{s}\left\{s \bar{y}_{1, k}(s)-y_{1,0}(0)-\mathscr{L}\left\{2 y_{2, k}\left(\frac{t}{2}\right)+y_{3, k}(t)+f_{1}(t)\right\}\right\}\right\} \\
&=\mathscr{L}^{-1}\left[\frac{-1}{s}\right]+\mathscr{L}^{-1}\left\{\frac{1}{s}\left\{\mathscr{L}\left\{2 y_{2, k}\left(\frac{t}{2}\right)+y_{3, k}(t)+f_{1}(t)\right\}\right\}\right\}, \\
& y_{2, k+1}(t)=y_{2, k}(t)-\mathscr{L}^{-1}\left\{\frac{1}{s}\left\{s \bar{y}_{2, k}(s)-y_{2,0}(0)+\mathscr{L}\left\{-2\left(y_{3, k}\left(\frac{t}{2}\right)\right)^{2}+f_{2}(t)\right\}\right\}\right\} \\
&=\mathscr{L}^{-1}\left\{\frac{1}{s}\left\{\mathscr{L}\left\{-2\left(y_{3, k}\left(\frac{t}{2}\right)\right)^{2}+f_{2}(t)\right\}\right\}\right\}, \\
& y_{3, k+1}(t)=y_{3, k}(t)-\mathscr{L} \mathscr{L}^{-1}\left\{\frac{1}{s}\left\{s \bar{y}_{3, k}(s)-y_{3,0}(0)-\mathscr{L}\left\{y_{2, k}(t)-y_{1, k}(t)+f_{3}(t)\right\}\right\}\right\} \\
&=\mathscr{L}^{-1}\left\{\frac{1}{s}\left\{\mathscr{L}\left\{y_{2, k}(t)-y_{1, k}(t)+f_{3}(t)\right\}\right\}\right\}, \quad \\
& \\
& y_{1,1}(t)=-2 t \sin \left(\frac{t}{2}\right)-4 \cos \left(\frac{t}{2}\right), \\
& y_{2,1}(t)=t \cos (t)-\sin (t)+t, \\
& y_{3,1}(t)=-t \sin (t)-\cos (t)+t+1,
\end{aligned}
$$

with the initial iterations above. Now, in order to obtain the first approximation $y_{1,1}(t), y_{2,1}$, and $y_{3,1}(t)$ of LVIA solution for (30)-(31), we put $k=0$ through (34) to get 
by using the first approximations $y_{1,1}(t), y_{2,1}(t)$, and $y_{3,1}(t)$ and then the second approximations $y_{i, 2}(t), i=1,2,3$, of LVIA solution for (30)-(31) which can be written in the form

$$
\begin{aligned}
y_{1,2(t)}= & 2 t\left(\cos \left(\frac{t}{2}\right)\right)^{2}-4 \sin \left(\frac{t}{2}\right) \cos \left(\frac{t}{2}\right)+t^{2}+4 \cos \left(\frac{t}{2}\right)-5 \\
y_{2,2}(t) & =\frac{1}{2} \cos \left(\frac{t}{2}\right) \sin \left(\frac{t}{2}\right) t^{2}-7 \cos \left(\frac{t}{2}\right) \sin \left(\frac{t}{2}\right)+12 t \sin \left(\frac{t}{2}\right)+16 \sin \left(\frac{t}{2}\right)+5 t\left(\cos \left(\frac{t}{2}\right)\right)^{2} \\
& -2 \cos \left(\frac{t}{2}\right) t^{2}-\frac{1}{4 t^{3}}-4 t \cos \left(\frac{t}{2}\right)-t^{2}+24 \cos \left(\frac{t}{2}\right)-\frac{9}{2 t}-24 \\
y_{3,2}(t) & =2\left(\cos \left(\frac{t}{2}\right)\right)^{2}-4 t \cos \left(\frac{t}{2}\right)+\frac{1}{2 t^{2}}+16 \sin \left(\frac{t}{2}\right)-3 t-2 .
\end{aligned}
$$

Using a similar approach, we get the $4^{\text {th }}$-order approximations $y_{i, 4}(t), i=1,2$, of the LVIA solution for (30) and (31) leading to the following results:

$$
\begin{aligned}
y_{1,3}(t)= & 8\left(\cos \left(\frac{t}{4}\right)\right)^{3} \sin \left(\frac{t}{4}\right)-8 \cos \left(\frac{t}{4}\right) \sin \left(\frac{t}{4}\right) t-4 \sin \left(\frac{t}{4}\right) t^{2}-4 \cos \left(\frac{t}{4}\right) \sin \left(\frac{t}{4}\right) \\
& -16 t \sin \left(\frac{t}{4}\right)+512 \sin \left(\frac{t}{4}\right)-\frac{1}{2}\left(\cos \left(\frac{t}{4}\right)\right)^{2} t^{2}-\frac{t^{4}}{64}-52\left(\cos \left(\frac{t}{4}\right)\right)^{2} \\
& -80 t \cos \left(\frac{t}{4}\right)-\frac{9}{4} t^{2}-192 \cos \left(\frac{t}{4}\right)-49 t+243 \\
y_{2,3}(t)= & -16\left(\cos \left(\frac{t}{4}\right)\right)^{3} \sin \left(\frac{t}{4}\right)+\frac{64(\cos (t / 4))^{2} \sin (t / 4) t}{3}-18 \cos \left(\frac{t}{4}\right) \sin \left(\frac{t}{4}\right) t^{2}+4 \sin \left(\frac{t}{4}\right) t^{3} \\
& +24 \cos \left(\frac{t}{4}\right) \sin \left(\frac{t}{4}\right) t-48 \sin \left(\frac{t}{4}\right) t^{2}+1704 \cos \left(\frac{t}{4}\right) \sin \left(\frac{t}{4}\right)-\frac{1984 t \sin (t / 4)}{3}+3072 \sin \left(\frac{t}{4}\right) \\
& +8 t\left(\cos \left(\frac{t}{4}\right)\right)^{4}-\frac{t^{5}}{160}+\frac{3}{16} t^{4}+\frac{1792(\cos (t / 4))^{3}}{9}-336 t\left(\cos \left(\frac{t}{4}\right)\right)^{2}+80 \cos \left(\frac{t}{4}\right) t^{2}-\frac{8}{3} t^{3} \\
& +48\left(\cos \left(\frac{t}{4}\right)\right)^{2}-768 t \cos \left(\frac{t}{4}\right)-3 t^{2}-\frac{9472 \cos (t / 4)}{3}-93 t+\frac{26192}{9}, \\
& +\frac{32768(\cos (t / 8))^{2} \sin (t / 8)}{27}-\frac{512 t(\cos (t / 8))^{3}}{9}-192 t\left(\cos \left(\frac{t}{8}\right)\right)^{8}+384 t\left(\cos \left(\frac{t}{8}\right)\right)^{6} \\
y_{3,3}(t)= & 2 \cos \left(\frac{t}{2}\right) \sin \left(\frac{t}{2}\right) t-4 \sin \left(\frac{t}{2}\right) t^{2}-8 t \sin \left(\frac{t}{2}\right)+120 \sin \left(\frac{t}{2}\right)-\frac{1}{2}\left(\cos \left(\frac{t}{2}\right)\right)^{2} t^{2} \\
& -\frac{1}{16} t^{4}-\frac{2}{3} t^{3}+5\left(\cos \left(\frac{t}{2}\right)\right)^{2}-40 t \cos \left(\frac{t}{2}\right)-\frac{5}{4} t^{2}-48 \cos \left(\frac{t}{2}\right)-19 t+43, \\
t_{1,4}(t)= & 142847+\frac{53788 t}{9}-\frac{297 t^{2}}{2}-t^{3}+2 \cos \left(\frac{t}{8}\right) \sin \left(\frac{t}{8}\right) t^{2}-1152 \cos \left(\frac{t}{8}\right) \sin \left(\frac{t}{8}\right) t \\
& -28\left(\cos \left(\frac{t}{8}\right)\right)^{2} t^{2}+512\left(\cos \left(\frac{t}{8}\right)\right)^{7} \sin \left(\frac{t}{8}\right)-768\left(\cos \left(\frac{t}{8}\right)\right)^{5} \sin \left(\frac{t}{8}\right) \\
& \\
&
\end{aligned}
$$




$$
\begin{aligned}
& +64\left(\cos \left(\frac{t}{8}\right)\right)^{4} t^{2}-8 \cos \left(\frac{t}{8}\right) t^{3}-\frac{t^{5}}{128}-\frac{1}{3 t^{4}}-32\left(\cos \left(\frac{t}{8}\right)\right)^{7} \sin \left(\frac{t}{8}\right) t^{2} \\
& +48\left(\cos \left(\frac{t}{8}\right)\right)^{5} \sin \left(\frac{t}{8}\right) t^{2}-20\left(\cos \left(\frac{t}{8}\right)\right)^{3} \sin \left(\frac{t}{8}\right) t^{2}-896\left(\cos \left(\frac{t}{8}\right)\right)^{3} \sin \left(\frac{t}{8}\right) t \\
& -704\left(\cos \left(\frac{t}{8}\right)\right)^{3} \sin \left(\frac{t}{8}\right)+512 \sin \left(\frac{t}{8}\right) t^{2}+1248 \sin \left(\frac{t}{8}\right) \cos \left(\frac{t}{8}\right)-9216 t \sin \left(\frac{t}{8}\right) \\
& -112 t\left(\cos \left(\frac{t}{8}\right)\right)^{4}-176 t\left(\cos \left(\frac{t}{8}\right)\right)^{2}+192 \cos \left(\frac{t}{8}\right) t^{2}+\frac{40448 t \cos (t / 8)}{3} \\
& -3648\left(\cos \left(\frac{t}{8}\right)\right)^{4}-122880 \cos \left(\frac{t}{8}\right)-\frac{4210688 \sin (t / 8)}{27}-16320\left(\cos \left(\frac{t}{8}\right)\right)^{2} \text {, } \\
& y_{2,4}(t)=-\frac{160053 t}{32}-203 t^{2}-\frac{12345 t^{3}}{64}+\frac{163 t(\cos (t / 4))^{4}}{4}-\frac{1}{32}\left(\cos \left(\frac{t}{4}\right)\right)^{3} \sin \left(\frac{t}{4}\right) t^{4} \\
& -\frac{\cos (t / 4) \sin (t / 4) t^{6}}{256}-\frac{1}{12} \cos \left(\frac{t}{4}\right) \sin \left(\frac{t}{4}\right) t^{5}+\frac{51(\cos (t / 4))^{3} \sin (t / 4) t^{2}}{8} \\
& -\frac{200(\cos (t / 4))^{2} \sin (t / 4) t^{3}}{9}+\frac{293 \cos (t / 4) \sin (t / 4) t^{4}}{64}-64\left(\cos \left(\frac{t}{4}\right)\right)^{2} \sin \left(\frac{t}{4}\right) t^{2} \\
& +\frac{227}{6} \cos \left(\frac{1}{4 t}\right) \sin \left(\frac{1}{4 t}\right) t^{3}-15840 \cos \left(\frac{1}{4 t}\right) \sin \left(\frac{1}{4 t}\right) t-\frac{35}{768} t^{6} \\
& -\frac{57595 \cos (t / 4) \sin (t / 4) t^{2}}{16}+\frac{53440(\cos (t / 4))^{2} \sin (t / 4) t}{27}+8288 \sin \left(\frac{t}{4}\right) t^{2}-\frac{2723 t^{5}}{3840}-\frac{49 t^{4}}{48} \\
& \frac{472960(\cos (t / 4))^{3}}{81}+1175\left(\cos \left(\frac{t}{4}\right)\right)^{2} t^{2}-\frac{363(\cos (t / 4))^{3} \sin (t / 4)}{4}+\frac{5780 \sin (t / 4) t^{3}}{9} \\
& \frac{879851 \sin (t / 4) \cos (t / 4)}{8}-\frac{1288192 t \sin (t / 4)}{27}-\frac{126907 t(\cos (t / 4))^{2}}{4}+\frac{21080 \cos (t / 4) t^{2}}{3} \\
& 45312 t \cos \left(\frac{t}{4}\right)-77760\left(\cos \left(\frac{t}{4}\right)\right)^{2}-\frac{2923648 \cos (t / 4)}{27}-\frac{1298176 \sin (t / 4)}{9} \\
& \frac{1}{16} \cos \left(\frac{t}{4}\right) t^{6}+\frac{19 \cos (t / 4) t^{5}}{12}-\frac{11}{4} \sin \left(\frac{t}{4}\right) t^{5}-\frac{313 \cos (t / 4) t^{4}}{6}-\frac{184 \sin (t / 4) t^{4}}{3} \\
& -\frac{2908 \cos (t / 4) t^{3}}{3}-\frac{t^{8}}{6144}-\frac{13 t^{7}}{4608}-\frac{t^{9}}{294912}+\frac{14596544}{81}+\frac{2}{3}\left(\cos \left(\frac{t}{4}\right)\right)^{3} t^{4} \\
& -\frac{2720(\cos (t / 4))^{3} t^{2}}{9}+\frac{1553(\cos (t / 4))^{2} t^{3}}{8}-\frac{5}{8}\left(\cos \left(\frac{t}{4}\right)\right)^{4} t^{3}-\frac{5(\cos (t / 4))^{2} t^{5}}{64} \\
& 13\left(\cos \left(\frac{t}{4}\right)\right)^{3} t^{3}-\frac{3}{2}\left(\cos \left(\frac{t}{4}\right)\right)^{2} t^{4}-\frac{1600(\cos (t / 4))^{3} t}{3}+\frac{17920(\cos (t / 4))^{2} \sin (t / 4)}{9}, \\
& y_{3,4}(t)=\cos \left(\frac{t}{4}\right) \sin \left(\frac{t}{4}\right) t^{2}+\frac{8192(\cos (t / 4))^{2} \sin (t / 4)}{27}-800 \cos \left(\frac{t}{4}\right) \sin \left(\frac{t}{4}\right) t+512 \sin \left(\frac{t}{4}\right) t^{2} \\
& .320 \cos \left(\frac{t}{4}\right) \sin \left(\frac{t}{4}\right)-4160 t \sin \left(\frac{t}{4}\right)-\frac{1025024 \sin (t / 4)}{27}-\frac{t^{6}}{960}+\frac{13 t^{5}}{320}+24\left(\cos \left(\frac{t}{4}\right)\right)^{4} \\
& -\frac{256(\cos (t / 4))^{3} t}{9}+36\left(\cos \left(\frac{t}{4}\right)\right)^{2} t^{2}-16 \cos \left(\frac{t}{4}\right) t^{3}-\frac{2}{3} t^{4}-60 t\left(\cos \left(\frac{t}{4}\right)\right)^{2}+176 \cos \left(\frac{t}{4}\right) t^{2} \\
& -\frac{1}{6} t^{3}-5016\left(\cos \left(\frac{t}{4}\right)\right)^{2}+\frac{20032 t \cos (t / 4)}{3}-\frac{245 t^{2}}{2}-26880 \cos \left(\frac{t}{4}\right)+\frac{24725 t}{9}+31872 \text {. }
\end{aligned}
$$




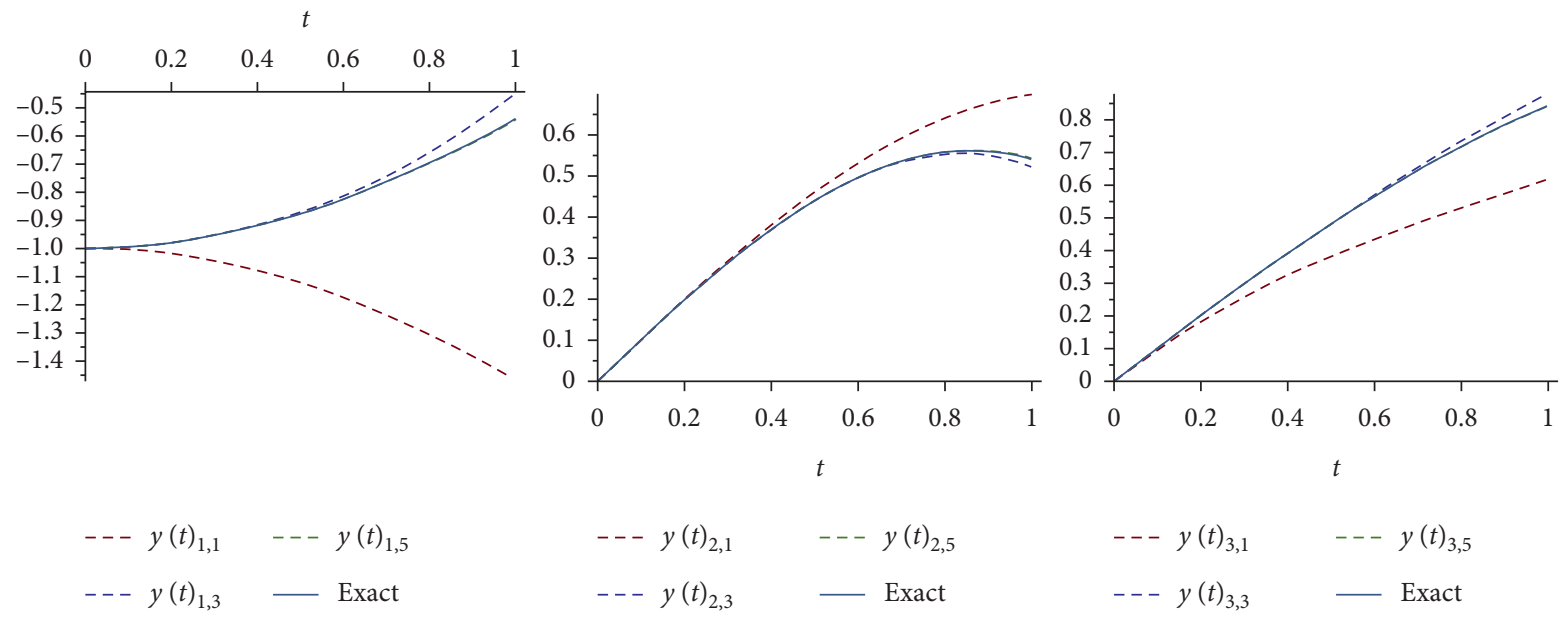

FIgURE 5: Plots of the LVIA solutions $y_{i, k}(t), i=1,2,3, k=1,3,5$, respectively, and the exact solution $y_{i}(t), i=1,2,3$ of Example 5 on [0, 1 , where $y_{i}(t)$ is represented by a straight line and $y_{i, k}(t)$ is represented by a dashed line.

TABLE 6: Error analysis and CPU times.

\begin{tabular}{|c|c|c|c|c|c|}
\hline$t$ & Absolute error $\mathrm{Ext}_{2}^{4}$ & relative error $\mathrm{Rel}_{2}^{4}$ & consecutive error $\mathrm{Con}_{2}^{4}$ & residual error $\operatorname{Res}_{3}^{4}(t)$ & $\mathrm{CPU}$ \\
\hline \multicolumn{6}{|c|}{ Error analysis and CPU times of $y_{1}(t)$ for Example 5 on $[0,1]$} \\
\hline 0.2 & $5.77 \times 10^{-5}$ & $5.89 \times 10^{-5}$ & $7.87 \times 10^{-5}$ & $1.24 \times 10^{-4}$ & 0.058 \\
\hline 0.4 & $4.16 \times 10^{-5}$ & $4.52 \times 10^{-5}$ & $2.27 \times 10^{-3}$ & $1.37 \times 10^{-3}$ & 0.057 \\
\hline 0.6 & $6.72 \times 10^{-4}$ & $3.69 \times 10^{-3}$ & $1.08 \times 10^{-2}$ & $6.66 \times 10^{-3}$ & 0.051 \\
\hline 0.8 & $2.57 \times 10^{-3}$ & $3.69 \times 10^{-3}$ & $3.41 \times 10^{-2}$ & $2.23 \times 10^{-2}$ & 0.049 \\
\hline 1.0 & $7.43 \times 10^{-3}$ & $1.38 \times 10^{-2}$ & $8.30 \times 10^{-2}$ & $5.71 \times 10^{-2}$ & 0.047 \\
\hline \multicolumn{6}{|c|}{ Error analysis and CPU times of $y_{2}(t)$ for Example 5 on $[0,1]$} \\
\hline 0.2 & $1.27 \times 10^{-5}$ & $4.86 \times 10^{-4}$ & $7.34 \times 10^{-6}$ & $7.27 \times 10^{-6}$ & 0.054 \\
\hline 0.4 & $6.88 \times 10^{-5}$ & $3.91 \times 10^{-3}$ & $1.56 \times 10^{-4}$ & $6.16 \times 10^{-5}$ & 0.061 \\
\hline 0.6 & $3.17 \times 10^{-5}$ & $1.39 \times 10^{-2}$ & $1.30 \times 10^{-3}$ & $4.43 \times 10^{-4}$ & 0.053 \\
\hline 0.8 & $1.18 \times 10^{-4}$ & $3.64 \times 10^{-2}$ & $5.49 \times 10^{-3}$ & $1.95 \times 10^{-3}$ & 0.050 \\
\hline 1.0 & $9.11 \times 10^{-4}$ & $8.53 \times 10^{-2}$ & $1.65 \times 10^{-2}$ & $6.06 \times 10^{-3}$ & 0.048 \\
\hline \multicolumn{6}{|c|}{ Error analysis and CPU times of $y_{3}(t)$ for Example 5 on $[0,1]$} \\
\hline 0.2 & $9.33 \times 10^{-6}$ & $4.70 \times 10^{-5}$ & $7.87 \times 10^{-5}$ & $2.74 \times 10^{-4}$ & 0.077 \\
\hline 0.4 & $1.88 \times 10^{-4}$ & $4.84 \times 10^{-4}$ & $2.27 \times 10^{-3}$ & $2.38 \times 10^{-3}$ & 0.057 \\
\hline 0.6 & $1.49 \times 10^{-3}$ & $2.64 \times 10^{-3}$ & $1.08 \times 10^{-2}$ & $1.19 \times 10^{-2}$ & 0.057 \\
\hline 0.8 & $6.57 \times 10^{-3}$ & $9.15 \times 10^{-3}$ & $3.14 \times 10^{-2}$ & $3.97 \times 10^{-3}$ & 0.051 \\
\hline 1.0 & $2.08 \times 10^{-2}$ & $2.47 \times 10^{-2}$ & $8.30 \times 10^{-2}$ & $9.92 \times 10^{-2}$ & 0.049 \\
\hline
\end{tabular}

To clarify the convergence of $y_{i, k}(t)$ to $y_{i}(t), i=1,2,3$, with respect to the number of iterations of the solution, we present numerical solutions of Example 5 graphically, where Figure 5 shows the exact solutions $y_{i}(t), i=1,2,3$, and the approximate solution of LVIA for $y_{i, k}(t), i=1,2,3$ and $k=1,3,5$, respectively. We will test the accuracy of LVIA for Example 5 by using error analysis of $y_{i}(t), i=1,2,3$, $t \in[0,1]$ for (30)-(31) with a step size of 0.2 as well as comparison among the absolute errors, relative errors, consecutive errors residual errors, and computing times (CPU) of $4^{\text {th }}$-order approximate LVIA solutions shown in Table 6.

Example 6. Consider 3-dimensional nonlinear SMPDDEs [23]:

$$
\begin{aligned}
& y_{1}^{\prime}(t)=y_{2}^{2}(t), \\
& y_{2}^{\prime}(t)=\frac{1}{2} y_{1}\left(\frac{t}{2}\right), \quad t \geq 0, \\
& y_{3}^{\prime}(t)=e^{(5 / 2) t} y_{2}(t)+9 e^{2 t} y_{3}(t)\left(\frac{t}{3}\right),
\end{aligned}
$$

subject to the initial conditions:

$$
\begin{aligned}
& y_{1}(0)=1, \\
& y_{2}(0)=1, \\
& y_{3}(0)=0 .
\end{aligned}
$$

The exact solutions are $y_{1}(t)=e^{t}, y_{2}(t)=e^{(t / 2)}$, and $y_{3}(t)=t e^{3 t}$. By comparing between the absolute errors of 
TABLE 7: Comparison of (absolute error) $\operatorname{Ext}_{i}^{k}$ for Example 6.

\begin{tabular}{lcccccc}
\hline$t$ & \multicolumn{2}{c}{$y_{1,4}(t)$} & \multicolumn{2}{c}{$y_{2,4}(t)$} & & $y_{3,4}(t)$ \\
LDM [23] & LVIA & ADM [23] & LVIA & ADM [23] & 0 \\
\hline 0.0 & 0 & 0 & 0 & 0 & $1.39 \times 10^{-12}$ & $3.00 \times 10^{-9}$ \\
0.1 & $1.00 \times 10^{-9}$ & $2.31 \times 10^{-11}$ & 0 & $9.13 \times 10^{-11}$ & $4.30 \times 10^{-8}$ & $2.57 \times 10^{-11}$ \\
0.2 & $9.10 \times 10^{-8}$ & $1.59 \times 10^{-9}$ & $1.00 \times 10^{-9}$ & $2.08 \times 10^{-9}$ \\
0.3 & $1.06 \times 10^{-6}$ & $1.90 \times 10^{-8}$ & $1.60 \times 10^{-8}$ & $1.07 \times 10^{-9}$ & $3.00 \times 10^{-9}$ & $3.00 \times 10^{-8}$ \\
0.4 & $6.03 \times 10^{-6}$ & $1.14 \times 10^{-7}$ & $9.10 \times 10^{-8}$ & $6.14 \times 10^{-9}$ & $5.05 \times 10^{-7}$ & $2.14 \times 10^{-7}$ \\
0.5 & $2.34 \times 10^{-5}$ & $4.60 \times 10^{-7}$ & $3.52 \times 10^{-7}$ & $2.40 \times 10^{-8}$ & $2.87 \times 10^{-6}$ & $1.04 \times 10^{-6}$ \\
0.6 & $7.084 \times 10^{-5}$ & $1.47 \times 10^{-6}$ & $1.06 \times 10^{-6}$ & $7.36 \times 10^{-8}$ & $1.01 \times 10^{-5}$ & $3.94 \times 10^{-6}$ \\
0.7 & $1.81 \times 10^{-4}$ & $3.92 \times 10^{-6}$ & $2.69 \times 10^{-6}$ & $1.90 \times 10^{-7}$ & $3.16 \times 10^{-5}$ & $1.26 \times 10^{-5}$ \\
0.8 & $4.10 \times 10^{-4}$ & $9.28 \times 10^{-6}$ & $6.03 \times 10^{-6}$ & $4.35 \times 10^{-7}$ & $8.86 \times 10^{-5}$ & $1.36 \times 10^{-5}$ \\
0.9 & $8.45 \times 10^{-4}$ & $2.00 \times 10^{-5}$ & $1.23 \times 10^{-5}$ & $9.03 \times 10^{-7}$ & $2.28 \times 10^{-4}$ & $9.26 \times 10^{-5}$ \\
1.0 & $1.62 \times 10^{-3}$ & $4.00 \times 10^{-5}$ & $2.33 \times 10^{-5}$ & $1.74 \times 10^{-6}$ & $5.40 \times 10^{-4}$ & $2.22 \times 10^{-4}$ \\
\hline
\end{tabular}
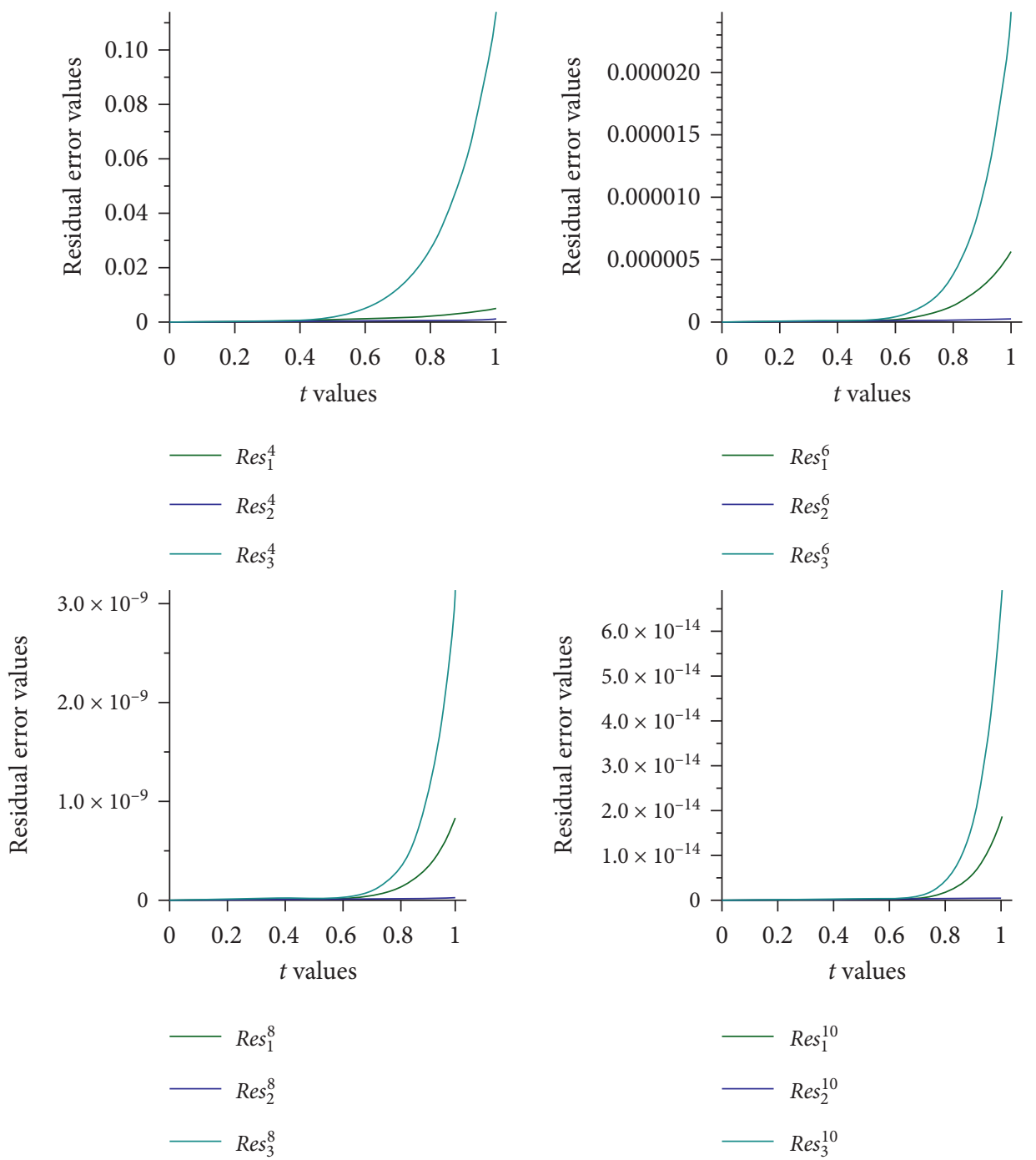

FIgURE 6: The residual error $\operatorname{Res}_{i}^{k}(t), i=1,2,3$ of LVIA solutions of (38) at $k=4,6,8,10$, respectively, for Example 6 .

LVIA for four iterations approximations and Adomian decomposition method (ADM) [23] which are shown in Table 7 , it is obvious that, from this table, the approximate solutions are identical to the exact solutions for all possible values of $t$ in $[0,1]$. Figure 6 shows that the residual error is the difference between both sides of equation (38), indicating that the two sides are identical with the increase of $k$, which shows the efficiency of the proposed method. 


\section{Conclusion}

The main objective of this paper is to adapt Laplace variational iteration algorithm to investigate SMPDDEs. This work aims to show the power of LVIA by reducing the numerical calculation which does not need any perturbations, discretization, and other restrictive hypotheses which may change the framework for the problem being solved. LVIA gives fast convergent successive approximations. We approve that the accuracy for LVIA gives it numerous wider applicability. Additionally, the results obtained by LVIA have the ability to be successful and furthermore helpful in linear and nonlinear cases since they do not require much computational work. It is obvious from the tables and figures that the absolute errors relief as $k$ rises. In any case, the numerical results show that LVIA is a reliable technique for SMPDDEs. LVIA is a relatively new approach to provide a symbolic approximation, in a rapidly convergent series by using the initial conditions only and without tedious calculations which show the solution in a closed form, for linear and nonlinear problems and it changes SMPDDEs to the recurrence sequence of functions. Though the LVIA leads to convergent solutions for a wider region as in Examples 1 and 2, but in certain situations, the series converges very quickly in a very small zone or neighborhood of the boundary points, and it has a very slow convergence rate in the wider and/or outer zone.

\section{Abbreviations}

VIA: Variational iteration algorithm

LVIA: Laplace variational iteration algorithm

DDEs: $\quad$ Delay differential equations

SMPDDEs: System of multipantograph delay differential equations.

\section{Data Availability}

The datasets used and/or analyzed during the current study are available from the corresponding author on reasonable request.

\section{Conflicts of Interest}

The authors declare no conflicts of interest with respect to the research and authorship of this article.

\section{Authors' Contributions}

The authors have contributed to each part of this paper. They read and approved the final manuscript.

\section{Acknowledgments}

This paper was supported financially by the Academy of Scientific Research and Technology (ASRT), Egypt, under initiatives of Science Up Faculty of Science (Grant No. 6639). ASRT is the second affiliation of this research.

\section{References}

[1] M. Arnold and B. Simeon, "Pantograph and catenary dynamics: a benchmark problem and its numerical solution," Applied Numerical Mathematics, vol. 34, no. 4, pp. 345-362, 2000.

[2] H. Jafari, M. Mahmoudil, and M. H. Noori Skandar, "A new numerical method to solve pantograph delay differential equations with convergence analysis," Advances in Difference Equations, vol. 2021, Article ID 129, 2021.

[3] S. Davaeifar and J. Rashidinia, "Solution of a system of delay differential equations of multi pantograph type," Journal of Taibah University for Science, vol. 11, no. 6, pp. 1141-1157, 2017.

[4] A. Isah, C. Phang, and P. Phang, "Collocation method based on Genocchi operational matrix for solving generalized fractional pantograph equations," International Journal of Differential Equations, vol. 2017, no. 7-8, 10 pages, Article ID 2097317, 2017.

[5] M. S. M. Bahgat, "Approximate analytical solution of the linear and nonlinear multi-pantograph delay differential equations," Physica Scripta, vol. 95, Article ID 055219, 2020.

[6] E. H. Doha, A. H. Bhrawy, and R. M. Hafez, "Numerical algorithm for solving multi-pantograph delay equations on the half-line using Jacobi rational functions with convergence analysis," Acta Mathematicae Applicatae Sinica, English Series, vol. 33, no. 2, pp. 297-310, 2017.

[7] A. Isah and C. Phang, "Operational matrix based on Genocchi polynomials for solution of delay differential equations," Ain Shams Engineering Journal, vol. 9, no. 4, pp. 2123-2128, 2018.

[8] T. Mehdi and D. Mehdi, "On the convergence of He's variational iteration method," Journal of Computational and Applied Mathematics, vol. 207, pp. 121-128, 2007.

[9] M. Torvattanabun and S. Koonprasert, "Convergence of the variational iteration method for solving a first-order linear system of PDEs with constant coefficients," Thai Journal of Mathematics, vol. 2009, pp. 1-13, 2009.

[10] O. Abu Arqub, M. Al-Smadi, and S. Momani, "Application of reproducing kernel method for solving nonlinear fredholmvolterra integrodifferential equations," Abstract and Applied Analysis, vol. 2012, Article ID 839836, 16 pages, 2012.

[11] R. Abu-Gdairi and M. H. Al-Smadi, "An efficient computational method for 4th-order boundary value problems of Fredholm IDEs," Applied Mathematical Sciences, vol. 7, pp. 4761-4774, 2013.

[12] B. Batiha, M. S. Noorani, and I. Hashim, "Approximate analytical solution of the coupled sine-Gordon equation using the variational iteration method," Physica Scripta, vol. 76, p. 4458, 2007.

[13] O. D. Makinde and R. J. Moitsheki, "On nonperturbative techniques for thermal radiation effect on natural convection past a vertical plate embedded in a saturated porous medium," Mathematical Problems in Engineering, vol. 2008, Article ID 689074, 11 pages, 2008.

[14] M. Al-Smadi, O. Abu Arqub, and A. El-Ajou, "A numerical iterative method for solving systems of first-order periodic boundary value problems," Journal of Applied Mathematics, vol. 2014, Article ID 135465, 10 pages, 2014.

[15] R. J. Moitsheki and O. D. Makinde, "Application of Lie point symmetry and Adomain decomposition methods to thermal storage diffusion equations," Journal of Physics: Conference Series, vol. 128, Article ID 012058, 2008.

[16] M. Al-Smadi, O. Abu Arqub, and S. Momani, "A computational method for two-point boundary value problems of 
fourth-order mixed integrodifferential equations," Mathematical Problems in Engineering, vol. 2013, Article ID 832074, 10 pages, 2013.

[17] Z. Altawallbeh, M. H. Al-Smadi, and R. Abu-Gdairi, "Approximate solution of second-order integrodifferential equation of Volterra type in RKHS method," International Journal of Mathematical Analysis, vol. 7, no. 44, pp. 21452160, 2013.

[18] O. Anwar Bég, M. M. Rashidi, T. A. Bég, and M. Asadi, "Homotopy analysis of transient magneto-biofluid dynamics of micropolar squeeze film in a porous medium: a model for magneto-bio-rheological lubrication," Journal of Mechanics in Medicine and Biology, vol. 12, pp. 1-12, 2012.

[19] I. Komashynska, M. Al-Smadi, A. Al-Habahbeh, and A. Ateiwi, "Analytical approximate solutions of systems of multipantograph delay differential equations using residual power-series method," Australian Journal of Basic and Applied Sciences, vol. 8, no. 10, pp. 664-675, 2014.

[20] M. E. Salih, "Exact solution of multi-pantograph equation using differential transform method," International Journal of Computational Science and Mathematics, vol. 6, no. 1, pp. 61-68, 2014.

[21] B. Aminu and N. Maan, "Analytical algorithm for systems of neutral delay differential equations," Journal of Applied Mathematics, vol. 2019, pp. 753-768, 2019.

[22] S. Widatalla and M. A. Koroma, "Approximation algorithm for a system of pantograph equations," Journal of Applied Mathematics, vol. 2012, Article ID 714681, 9 pages, 2012.

[23] K. S. Rostam and M. R. Botan, "Adomian decomposition method for solving system of delay differential equation," Ustralian Journal of Basic and Applied Sciences, vol. 4, no. 8, pp. 3613-3621, 2010. 